\title{
Complex Regional Pain Syndrome (CRPS type-1) in an Adolescent Following Extravasation of Dextrose Containing Fluid-an Underdiagnosed Case
}

Department of Anesthesiology and Critical Care, BPKIHS, Dharan, Nepal, *Department of Anesthesiology, North Eastern Indira Gandhi Regional Institute of Health and Medical Sciences, Shillong, India

Asish Subedi, MD, Balkrishna Bhattarai, MD, Binay K Biswas, MD*, and Sindhu Khatiwada, MD

Due to its complex pathophysiology and wide spectrum of clinical manifestations, the diagnosis of CRPS is often missed in the early stage by primary care physicians. After being treated by a primary care physician for 5 months for chronic cellulitis, a 16-year-old girl was referred to our hospital with features of type-1 CRPS of the right upper extremity. Inability to diagnose early caused prolonged suffering to the girl with all the consequence of CRPS. The patient responded well with marked functional recovery from multimodal therapy. Ability to distinguish CRPS from other pain conditions, referral for specialty care at the appropriate time and full awareness of this condition and its clinical features among various healthcare professionals are essential in reducing patient suffering and stopping its progression towards difficult-to-treat situations. (Korean J Pain 2011; 24: 112-114)

Key Words:

adolescent, cellulitis, CRPS type-1, misdiagnosed.

Complex regional pain syndrome (CRPS) type-1 is a painful and disabling chronic neuropathic disorder, usually precipitated by minor trauma in the extremities. Early recognition of the disease and its treatment can alter the course of the disease. We describe a patient who had delayed diagnosis of CRPS type-1 because of unawareness of this condition among primary care physicians and its subsequent successful management by specialty care. The purpose of this paper is to create awareness among non-specialist practitioners who often see patients with CRPS in the early stage of the disease, so as to distinguish it from other pain conditions and to initiate timely appropriate intervention to halt disease progression.

\section{CASE REPORT}

A 16-year-old female patient was referred to our pain clinic from the orthopedic department with persistent pain and swelling of the right forearm. The patient's history revealed her being admitted to a local hospital with urinary tract infection and receiving an infusion of $5 \%$ dextrose with $0.9 \%$ saline through an IV cannula placed on the right

Received February 21, 2011. Revised April 18, 2011. Accepted April 25, 2011.

Correspondence to: Asish Subedi, MD

Department of Anesthesiology and Critical Care, BP Koirala Institute of Health Sciences, Dharan, Nepal

Tel: +025-525555 \# 2012, Fax: +977-25-520251, E-mail: subediasish@yahoo.com

(c) This is an open-access article distributed under the terms of the Creative Commons Attribution Non-Commercial License (http:// creativecommons.org/licenses/by-nc/3.0/), which permits unrestricted non-commercial use, distribution, and reproduction in any medium, provided the original work is properly cited.

Copyright (c) The Korean Pain Society, 2011 
dorsum of the hand five months previous. Later, extravasation of fluid in the surrounding tissue occurred leading to pain and swelling that did not subside but kept increasing. The general practitioner's initial diagnosis was cellulitis. She was treated with analgesics and antibiotics followed by local incision and drainage. Despite symptomatic treatment and surgical intervention, the symptoms further worsened leaving surgical scars (Fig. 1). The primary care physician managed conservatively for almost 5 months before she presented to our hospital. Clinical examination revealed a tender, shinny, swollen limb with allodynia and stiff joints with no evidence of nerve injury suggestive of CRPS type-1. Doppler study ruled out any possibility of vascular disease. A diagnostic stellate ganglion block (SGB) improved the numerical pain rating score (0-10) from 7 to 2 with enhancement of limb mobility. A multimodal approach of treatment including oral medications (gabapentin, amitryptyline, tramadol), a series of SGB with bupivacaine, and limb physiotherapy were initiated. At 6 months follow-up, pain and swelling subsided drastically with marked functional recovery (Fig. 2).

\section{DISCUSSION}

Trauma is the most common precipitating event of CRPS type-1, the majority following minor insult to an extremity. The inciting event in our case was inadvertent extravasations of a dextrose solution into tissues sur-

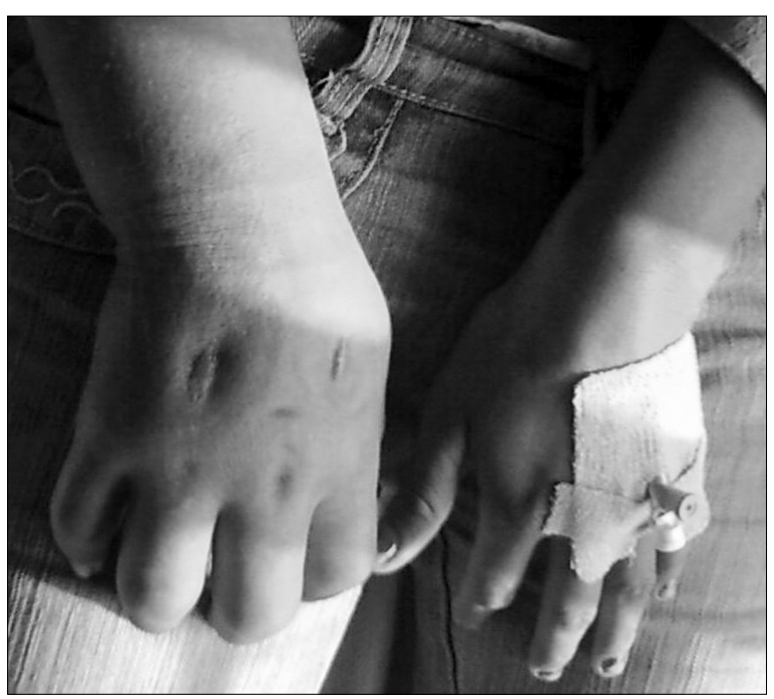

Fig. 1. Edematous and limited movement of right hand with scar marks. rounding the IV cannula. These extravasations to the interstitial space may have led to an inflammatory response resulting in edema, redness and pain. Rapid swelling and compression of the surrounding tissue may also cause acute limb compartment syndrome [1], reducing tissue perfusion. Swelling, inflammation, tenderness and skin changes have been reported to confound diagnosis of CRPS following snake bite also [2]. These mechanisms' roles cannot be ignored in our case as the current understanding of CRPS pathophysiology involves inflammation and hypoxia along with autonomic and somatic nervous system involvement [3]. However, it is difficult or even impossible to demonstrate a causal relationship between these clinical conditions and CRPS development.

Due to its wide range of precipitating factors and pathophysiologic complexity, CRPS diagnosis is based primarily on established clinical criteria including allodynia, temperature and skin color changes, edema, motor dysfunction and trophic changes [4]. Although special investigations, such as autonomic function tests and bone scans, support the diagnosis of CRPS, these diagnostic modalities add little to the overall accuracy of diagnosing the disease when compared with clinical criteria [5]. Moreover, availability of such modalities is unlikely in every clinical setting. Hence, distinguishing CRPS type-1 clinically from other pain conditions, such as undiagnosed deep vein thrombosis, cellulitis and vasculitis, can be challenging even to specialist pain physicians [6]. The clinical course of CRPS progresses through three stages: acute inflammatory followed by dystrophy, ultimately leading to structural atrophy. However, these stages may overlap with less distinctness [7]. In the acute stage, all classical signs and symptoms of inflammation are present, similar

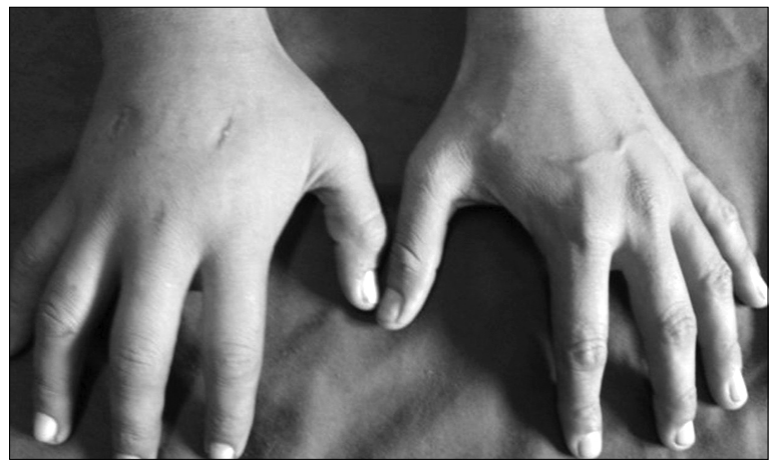

Fig. 2. Significant improvement of right hand seen after the therapy. 
to that of cellulitis. Moreover, in the early course of the disease, all features may not be present making diagnosis difficult [8]. Adolescents are less likely to have overt autonomic signs associated with CRPS, such as hyperhidrosis and trophic changes, compared to adults $[7,9]$. This may also lead to improper diagnosis.

With a multimodal treatment approach to CRPS, wide ranges of non-pharmacological therapeutic modalities, including intravenous regional blockade, sympathetic blockade and spinal cord stimulation, have been reported with varying results $[10,11]$. In our case, use of SGB combined with oral medications and limb physiotherapy not only served as therapy, but also contributed to confirming the diagnosis. It is interesting to note that although no special equipment or testing is required for diagnosis of CRPS type-1, an average of 30 months pass and 4.8 different physicians are visited before patients with CRPS are referred to a pain centre even in well-established centres $[12,13]$. These data show lack of disease awareness among general practitioners who often encounter such patients in the early stage, as in our case.

This case highlights that CRPS often remains underdiagnosed in the hands of non-specialist physicians, particularly if the patient presents with various inflammatory features such as cellulitis, arthritis, etc. Primary care physicians are often the first group of care providers to whom patients go for consultation. Disease awareness among practitioners, its early recognition, appropriate therapy at the beginning and referral to a pain centre shall prevent unnecessary suffering from CRPS and also may prevent the incidence of its development in the community.

\section{REFERENCES}

1. Doellman D, Hadaway L, Bowe-Geddes LA, Franklin M,
LeDonne J, Papke-O'Donnell $L$, et al. Infiltration and extravasation: update on prevention and management. J Infus Nurs 2009; 32: 203-11.

2. Bhattarai B, Shrestha BP, Rahman TR, Sharma SK, Tripathi M. Complex regional pain syndrome (CRPS) type-1 following snake bite: a case report. Nepal Med Coll J 2008; 10: 278-80.

3. de Mos M, Sturkenboom MC, Huygen FJ. Current understandings on complex regional pain syndrome. Pain Pract 2009; 9: 86-99.

4. Merskey H, Bogduk N. Classification of chronic pain: descriptions of chronic pain syndromes and definitions of pain terms. 2nd ed. Seattle, ASP Press. 1994, pp 210-7.

5. Oerlemans HM, Oostendorp RA, de Boo T, Perez RS, Goris RJ. Signs and symptoms in complex regional pain syndrome type 1/reflex sympathetic dystrophy: judgment of the physician versus objective measurement. Clin J Pain 1999; 15: 224-32.

6. Rho RH, Brewer RP, Lamer TJ, Wilson PR. Complex regional pain syndrome. Mayo Clin Proc 2002; 77: 174-80.

7. Hooshmand H. Chronic pain: reflex sympathetic dystrophy: prevention and management. Boca Raton, CRC Press. 1993.

8. Pittman DM, Belgrade MJ. Complex regional pain syndrome. Am Fam Physician 1997; 56: 2265-70.

9. Sherry DD. An overview of amplified musculoskeletal pain syndromes. J Rheumatol Suppl 2000; 58: 44-8.

10. Yoo HS, Nahm FS, Yim KH, Moon JY, Kim YS, Lee PB. Pregnancy in woman with spinal cord stimulator for complex regional pain syndrome: a case report and review of the literature. Korean J Pain 2010; 23: 266-9.

11. Tran de QH, Duong S, Bertini P, Finlayson RJ. Treatment of complex regional pain syndrome: a review of the evidence. Can J Anaesth 2010; 57: 149-66.

12. Allen G, Galer BS, Schwartz L. Epidemiology of complex regional pain syndrome: a retrospective chart review of 134 patients. Pain 1999; 80: 539-44.

13. Jänig W, Baron R. Experimental approach to CRPS. Pain 2004; 108: 3-7. 